\title{
Desempenho de índices quantitativos de seca na estimativa da produtividade de arroz de terras altas
}

\author{
Diego Simões Fernandes ${ }^{(1)}$, Alexandre Bryan Heinemann(1), Rosidalva Lopes Feitosa Paz ${ }^{(2)}$ \\ e André de Oliveira Amorim ${ }^{(2)}$
}

${ }^{(1)}$ EmbrapaArrozeFeijão, RodoviaGO462,Km12,ZonaRural,CEP75375-000SantoAntôniodeGoiás, GO.E-mail:diegosifer@cnpaf.embrapa.br,
alexbh@cnpaf.embrapa.br (2)Sistema de Meteorologia e Hidrologia do Estado de Goiás, Secretaria de Estado de Ciência e Tecnologia, Rua 82
s/no, Palácio Pedro L. Teixeira, 2o andar, Setor Sul, CEP 74088-900 Goiânia, GO. E-mail: dalvapaz@yahoo.com.br, amorim_go@yahoo.com.br

Resumo - O objetivo deste trabalho foi caracterizar a intensidade e a ocorrência de seca pelo uso de índices quantitativos, e avaliar a relação entre esses índices e os dados da série histórica da produtividade ajustada do arroz de terras altas da microrregião de Goiânia, GO. O ajuste da série histórica foi realizado para minimizar os efeitos da variabilidade climática da região e dos avanços tecnológicos sobre a produtividade. Foram avaliados os seguintes índices: severidade de seca de Palmer (PDSI); Z de Palmer (Z-index); o de anomalia de chuva (RAI); e o padronizado de precipitação (SPI). Os índices de seca foram analisados com uso da correlação de Pearson, número e frequência de ocorrência da seca e percentual de acerto dos índices em relação à produtividade ajustada. O RAI quantificou o maior número de eventos extremos de seca, enquanto o PDSI não estimou nenhum caso. O Z-index apresentou o maior percentual de acerto, em relação às variações ocorridas na produtividade ajustada. Em períodos com variações da produtividade ajustada maior que $300 \mathrm{~kg} \mathrm{ha}^{-1}$, Z-index, RAI e SPI apresentaram 78, 78 e $67 \%$ de percentuais de acerto, respectivamente. O Z-index teve o melhor desempenho na estimação da produtividade ajustada de arroz de terras altas.

Termos para indexação: Oryza sativa, deficiência hídrica, índice de anomalia da precipitação, índice de precipitação padronizada, índice de severidade da seca de Palmer, índice Z de Palmer.

\section{Performance of quantitative drought indices in the upland rice yield estimates}

Abstract - The objective of this work was to characterize the drought intensity and occurrence quantitative indices, and to evaluate relationship between these index and the upland rice yield historical data of Goiânia micro region, GO, Brazil. The historical data set was adjusted in order to minimize the effects of region's climatic variability and technological advances on crop yield. Four indices were evaluated: Palmer's drought severity index (PDSI), Palmer's Z index (Z-index), rainfall anomaly index (RAI), and the standardized precipitation index (SPI). Drought indices were analyzed trough Pearson's correlation, number and frequency of drought occurrence, and the index accuracy related to the upland rice adjusted yield data. RAI quantified the highest number of drought extreme occurences, while PDSI did not estimate any case. Z-index showed the highest accuracy in relation to the variations in the adjusted yield. In periods with rice adjusted yield variations higher than $300 \mathrm{~kg} \mathrm{ha}^{-1}$, the Z-index, RAI and SPI indices showed the highest accuracies with, 78, 78 and 67\%, respectively. The $Z$-index had the best performance in the estimation of upland rice adjusted yield.

Index terms: Oryza sativa, water deficit, rainfall anomaly index, standardized precipitation index, Palmer's drought severity index, Palmer's Z index.

\section{Introdução}

A seca é uma característica normal e periódica do clima, cujo início é difícil de detectar. De monitoramento complexo, ela causa prejuízos em diferentes setores da atividade humana (Quiring, 2009a). Secas ocorrem em qualquer região climática e são caracterizadas pelo deficit entre a precipitação pluvial e a evapotranspiração potencial. A gravidade da seca aumenta com a associação de temperatura do ar elevada, ventos fortes, baixa umidade do ar e com condições locais de solo, relacionadas, principalmente, à taxa de infiltração e retenção de água e à sua erodibilidade potencial (Freitas, 2005; Salas et al., 2005).

Segundo Redmond (2002), não há um conceito universal para definir a seca, o que permite que suas definições sejam dependentes da temática de abordagem. Heim (2002) classificou a seca em quatro diferentes categorias, de acordo com seus efeitos: meteorológica, agrícola, hidrológica e socioeconômica. 
Aseca meteorológica está relacionada somente ao deficit de precipitação pluvial e sua duração. A seca agrícola refere-se a situações com redução na quantidade de água disponível, nas diferentes camadas do solo ocupadas pelo sistema radicular, que afetariam o rendimento das culturas. O impacto desse tipo de seca depende da sua duração em relação à fase em que a cultura encontra-se (Park et al., 2005). Na seca hidrológica ocorrem períodos mais longos de deficit de precipitação pluvial e é observada após a seca meteorológica e a agrícola. Já a seca socioeconômica ocorre quando a deficiência de água começa a afetar a vida humana, e está vinculada aos outros três tipos de seca. Embora as secas estejam classificadas nesses quatro tipos, todas são originadas da deficiência de precipitação, que resulta na falta de água para o desenvolvimento de atividades ou para a sobrevivência dos seres vivos (Wilhite, 2003).

O Estado de Goiás destaca-se por sua vocação agrícola, e é responsável por $6,2,6,87$ e 1,52\% das produções brasileiras de soja, milho e arroz (Instituto Brasileiro de Geografia e Estatística, 2010). Entre essas culturas, o arroz de terras altas é considerado altamente sensível à deficiência hídrica (Pinheiro, 2006). A produtividade das culturas é diretamente influenciada pela ocorrência de secas agrícolas na região. Assim, há necessidade de se monitorar, por meio de ferramentas como os índices de seca, a intensidade e a ocorrência desses eventos.

Como existem diferentes índices de seca com distintas metodologias de cálculo, faz-se necessária uma avaliação de desempenho sobre qual índice apresenta melhores resultados a uma dada região. De acordo com Quiring (2009b), um único índice não pode representar todos os aspectos da seca, por isso é melhor utilizar uma abordagem multi-índice. Portanto, neste trabalho foram selecionados quatro índices, dos quais dois agrícolas, PDSI (Palmer, 1965) e Z-index (Palmer, 1965), e dois meteorológicos, SPI (McKee et al., 1993) e RAI (Rooy, 1965). No Brasil, Blain \& Brunini (2007a, 2007b) analisaram diferentes índices de seca (SPI, IPER, PDSI e PDSI modificado), para a região de São Paulo, e concluíram que o SPI pode ser utilizado para a análise de risco e o PDSI modificado para quantificar a seca meteorológica.

O objetivo deste trabalho foi caracterizar a intensidade e a ocorrência de seca pelo uso de índices quantitativos, e avaliar a relação entre esses índices e os dados da série histórica da produtividade ajustada do arroz de terras altas da microrregião de Goiânia, GO.

\section{Material e Métodos}

Para este trabalho, foi utilizada uma série histórica de dados diários das variáveis climáticas, precipitação pluvial e temperatura máxima e mínima do ar, coletados na estação meteorológica $\left(16^{\circ} 28^{\prime} 48^{\prime \prime} \mathrm{S}, 4^{\circ} 18^{\prime} 0^{\prime \prime} \mathrm{W}\right.$; altitude $823 \mathrm{~m}$ ), em Santo Antônio de Goiás, GO, de 1983 a 2008. A análise de consistência, como também os métodos de preenchimento, referentes às falhas nos dados climáticos dessa série histórica, seguiu metodologia apresentada por Heinemann et al. (2007).

A escolha de dois índices agrícolas e dois índices meteorológicos foi feita pelo fato de os índices meteorológicos serem de cálculos mais simples, com necessidade apenas de dados de precipitação pluvial (Guttman, 1998), enquanto os índices agrícolas, além da precipitação pluvial, necessitam de dados de temperatura máxima e mínima do ar.

No presente trabalho, são apresentadas somente as equações principais para o cálculo dos índices. Maiores detalhes sobre as equações e procedimentos dos cálculos estão descritos em Ntale \& Gan (2003), Loukas \& Vasiliadis (2004), Blain \& Brunini (2007a), Fernandes et al. (2009), Silva et al. (2009) e Macedo et al. (2010). Para o cálculo do balanço hídrico utilizado nos índices de seca agrícola (PDSI e Z-index), aplicou-se o método de Thornthwaite \& Matter (1955), tendo-se considerado as características hídricas de um Latossolo, que representa $46 \%$ dos solos do Estado de Goiás, com capacidade de armazenamento de $100 \mathrm{~mm}$ de água e profundidade efetiva de 1,0 m (Schaffert, 2000). Para os quatro índices, a escala de tempo considerada foi a mensal.

O PDSI foi obtido pela equação desenvolvida por Palmer (1965). Para o primeiro mês da série histórica dos dados climáticos, o valor do PSDI foi calculado considerando-se somente o segundo termo Z-index/3, da equação PDSI $=0,897 \times$ PDSI $_{\mathrm{i}-1}+(\mathrm{Z}$-index/3), em razão de não se ter o referencial do mês anterior, em que Z-index é o índice de anomalia de umidade de Palmer, para um respectivo mês e ano, obtido pela equação Z-index $=\left(\mathrm{P}_{\mathrm{i}}-\overline{\mathrm{P}}_{\mathrm{i}}\right) \mathrm{K}_{\mathrm{i}}$ (Dunkel, 2009), em que: $\mathrm{P}_{\mathrm{i}}$ é a soma da precipitação pluvial mensal para um determinado mês e ano; $\overline{\mathrm{P}}_{\mathrm{i}}$ é o valor de precipitação pluvial esperada para prover uma região com condições hídricas normais, para um determinado mês; $\mathrm{K}_{\mathrm{i}}$ é a constante de padronização para um determinado mês, obtida mensalmente por um período de calibração, conforme descrito em Palmer (1965), Blain \& Brunini (2007a) e Fernandes et al. (2009). 
A distribuição gama com dois parâmetros e a sua função de probabilidade cumulativa (Thom, 1958), é a mais comumente utilizada para o cálculo do SPI. O cálculo do SPI leva em consideração dados de precipitação pluvial ajustada por meio da distribuição gama e, posteriormente, transformada em uma distribuição normal que, por definição, apresenta sua média com valor zero e variância unitária (Wu et al., 2005). A equação,

$$
\mathrm{SPI}=\left\{ \pm \mathrm{t}-\left[\left(\mathrm{c}_{0}+\mathrm{c}_{1}+\mathrm{c}_{2} \mathrm{t}^{2}\right) /\left(1+\mathrm{d}_{1} \mathrm{t}+\mathrm{d}_{2} \mathrm{t}^{2}+\mathrm{d}_{3} \mathrm{t}^{3}\right)\right]\right\},
$$

descreve como foram obtidos os valores do SPI, em que: $\mathrm{t}$ é uma variável dependente da distribuição de probabilidade cumulativa; $\mathrm{c}_{0}, \mathrm{c}_{1}, \mathrm{c}_{2}, \mathrm{~d}_{1}, \mathrm{~d}_{2}$ e $\mathrm{d}_{3}$ são coeficientes constantes.

O cálculo do RAI (Rooy, 1965) é descrito pela equação,

$$
R A I= \pm\left[\left(P_{i}-P_{m}\right) /\left(\overline{P_{10}}-P_{m}\right)\right]
$$

em que: $\mathrm{P}_{\mathrm{m}}$ é a precipitação pluvial média mensal da série; $\overline{\mathrm{P}_{10}}$ é a precipitação pluvial média dos dez mais altos valores (anomalia positiva) ou dos dez mais baixos valores (anomalia negativa) da série.

Os dados históricos de produtividade da cultura de arroz de terras altas, dos anos agricolas 1983/1984 a 2005/2006, utilizados neste trabalho, pertencem à microrregião de Goiânia, onde se encontra o Município de Santo Antônio de Goiás. Esses dados são provenientes da base de dados Agrotec (Garagorry et al., 1997). Para essa microrregião, os dados históricos de produtividade do arroz de terras altas apresentam crescimento exponencial, em função das interações relacionadas à variabilidade climática da região e aos avanços tecnológicos. Assim, os efeitos da tendência tecnológica dessa série histórica de produtividade são minimizados e os dados são ajustados para o último ano com dados disponíveis de produtividade do ano de referência, na base de dados (Hollinger et al., 2001). No presente trabalho, o ano de referência foi 2006. Uma linha de tendência foi ajustada de acordo com os dados de produtividade observados, tendo-se utilizado o método não paramétrico de regressão polinomial "loess" (Cleveland, 1979). A linha obtida em função dos valores estimados pela regressão foi considerada como o avanço tecnológico. Os desvios relativos da regressão, que representam as variações climáticas, foram obtidos pela equação $\mathrm{RD}_{\mathrm{i}}^{\mathrm{n}}=(\mathrm{x}-\overline{\mathrm{y}}) / \overline{\mathrm{y}}$, em que: $\mathrm{RD}$ é o desvio relativo, $\mathrm{i}=1983$, e $\mathrm{n}=2006$; $\mathrm{x}$ é a produtividade observada $\left(\mathrm{kg} \mathrm{ha}^{-1}\right)$; e $\bar{y}$ é a produtividade estimada $\left(\mathrm{kg} \mathrm{ha}^{-1}\right)$ pela regressão. $\mathrm{O}$ ajuste dos dados de produtividade para o ano de 2006 foi calculado com base na equação $\mathrm{AY}_{\mathrm{i}}^{\mathrm{n}}=\left(\mathrm{RD}_{\mathrm{i}}^{\mathrm{n}}+1\right) \overline{\mathrm{Y}}$, em que: $\mathrm{AY}$ é a produtividade ajustada $\left(\mathrm{kg} \mathrm{ha}^{-1}\right)$; e $\overline{\mathrm{Y}}$ é a produtividade estimada $\left(\mathrm{kg} \mathrm{ha}^{-1}\right)$ pela regressão para o ano de 2006. Devese adicionar 1 ao RD, pois seus valores são sempre menores que 1 .

Os valores obtidos por meio dos índices de seca foram analisados utilizando-se correlação de Pearson, número de eventos de seca, frequência relativa dos resultados em função da classificação apresentada na Tabela 1, comparação com os dados de produtividade ajustados do arroz de terras altas e o número de acerto dos índices em relação a produtividade do arroz de terras altas.

Para analisar a tendência da série dos valores de índices de seca com os valores de produtividade ajustada do arroz de terras altas, somaram-se os valores dos índices referentes aos meses de novembro, dezembro, janeiro e fevereiro, que representam o principal período

Tabela 1. Classificação da umidade com base em índices de seca ${ }^{(1)}$.

\begin{tabular}{lcccc}
\hline Classe & PDSI & Z-index & SPI & RAI \\
\hline Extremamente úmido & $\geq 4,00$ & $\geq 4,00$ & $\geq 2,00$ & $\geq 4,00$ \\
Muito úmido & 3,00 a 3,99 & 3,00 a 3,99 & 1,00 a 1,99 & 3,00 a 3,99 \\
Moderadamente úmido & 2,00 a 2,99 & 2,00 a 2,99 & 0,50 a 0,99 & 2,00 a 2,99 \\
Pouco úmido & 0,50 a 1,99 & 0,50 a 1,99 & - & 0,50 a 1,99 \\
Próximo ao normal & 0,49 a $-0,49$ & 0,49 a $-0,49$ & 0,49 a $-0,49$ & 0,49 a $-0,49$ \\
Pouco seco & $-0,50$ a $-1,99$ & $-0,50$ a $-1,99$ & - & $-0,50$ a $-1,99$ \\
Moderadamente seco & $-2,00$ a $-2,99$ & $-2,00$ a $-2,99$ & $-0,50$ a $-0,99$ & $-2,00$ a $-2,99$ \\
Muito seco & $-3,00$ a $-3,99$ & $-3,00$ a $-3,99$ & $-1,00$ a $-1,99$ & $-3,00$ a $-3,99$ \\
Extremamente seco & $\leq-4,00$ & $\leq-4,00$ & $\leq 2,00$ & $\leq-4,00$
\end{tabular}

(1)PDSI, índice de severidade de seca de Palmer; Z-index, índice Z de Palmer; SPI, índice padronizado de precipitação; RAI, índice de anomalia de chuva. 
de crescimento e desenvolvimento da cultura na Região Centro-Oeste. Os valores somados dos índices de seca foram denominados índices acumulados.

Para analisar a variação na produtividade ajustada (diminuição/aumento), em razão dos períodos seco ou úmido, esses dados foram classificados em dois grupos: com variação menor ou igual a $2 \%$, em relação ao ano anterior; e com variação maior que $2 \%$. Para avaliar o número de acerto dos índices acumulados, em relação aos valores da produtividade ajustada, utilizou-se uma tabela de contingência, com as seguintes restrições: acerto, mês classificado como seco e diminuição da produtividade; erro, mês classificado como seco e ausência de diminuição da produtividade; negativo errado, mês classificado como úmido e diminuição da produtividade; e negativo correto, mês classificado como úmido e ausência de diminuição da produtividade. O número de acertos (Pr) foi calculado em função dos valores obtidos no "acerto" e "negativo correto" pela equação $\operatorname{Pr}=(\mathrm{Ac}-\mathrm{NC}) / \mathrm{NT}$, em que: Ac é o número de acertos; NC é o número de negativos corretos; e NT é o número total de amostras.

O número de acertos dos índices acumulados foi calculado para dois cenários diferentes: a série histórica total de dados de produtividade ajustada; e somente para os anos em que a produtividade ajustada variou mais que $300 \mathrm{~kg} \mathrm{ha}^{-1}$, em relação ao ano anterior. Esse valor corresponde à diminuição de aproximadamente $20 \%$ do menor valor da produtividade ajustada de arroz de terras altas.

\section{Resultados e Discussões}

A evolução da série temporal dos valores dos índices calculados permitiu observar os vários períodos secos durante os anos de 1983 a 2008, no Município de Santo Antônio de Goiás, GO (Figura 1). Na análise da série temporal do PDSI, segundo a classificação ilustrada na Tabela 1, observou-se que esse índice não classificou nenhum mês como extremamente seco; no entanto, classificou períodos muito secos, em 2 anos, moderadamente secos, em 5 anos, e pouco secos, em 18 anos (Figura 1 A). Foram observados, pelo PDSI, 126 meses com ocorrência de secas, das quais 4 meses muito secos, 21 moderadamente secos e 101 pouco secos (Figura $2 \mathrm{~A}$, linha). Os valores calculados por esse índice apresentaram defasagem em relação aos outros utilizados no presente trabalho (Figura $1 \mathrm{~B}, \mathrm{C}$ e D). No cálculo do PDSI, há dependência do índice relativo ao mês anterior, o que permite que essa defasagem resulte em baixa sensibilidade do índice em considerar os efeitos de um mês com precipitação próxima, porém inferior à média mensal histórica, caso o mês anterior tenha apresentado excedente significativo nos valores de precipitação. Essa defasagem também foi observada por Mckee et al. (1993) e Keyantash \& Dracup (2002), em razão da exagerada "memória" que o índice carrega. Esse índice indicou tendência de aumento do número de eventos de seca (Figura 1 A). A correlação de Pearson entre os dois índices quantificadores de seca agrícola, PDSI e Z-index, foi de 0,44 (Tabela 2). Apesar de ambos os índices integrarem o balanço hídrico em seus respectivos cálculos, essa baixa correlação pode ser justificada pelos problemas do PDSI supracitados.

O Z-index mostrou maior ocorrência de eventos de seca, em comparação ao PDSI. Isso pode ser observado na evolução da série temporal desse índice (Figura 1 B). Esse índice estimou períodos extremamente secos em 3 anos, muito secos em 7 anos, moderadamente secos em 14 anos e pouco secos em 26 anos. Foram observados 134 meses com ocorrência de eventos de seca estimados pelo Z-index, dos quais 3 meses foram classificados como extremamente secos, 8 meses como muito secos, 30 meses como moderadamente secos e 93 meses considerados pouco secos (Figura $2 \mathrm{~B}$, linha). Esse índice apresentou correlações de 0,91 e 0,87 com os índices SPI e RAI, respectivamente (Tabela 2). O SPI apresentou maior ocorrência no número de eventos extremos de seca, em comparação aos índices PDSI e Z-index. O SPI classificou períodos em 5 anos como de seca extrema, períodos em 17 anos como muito secos e períodos em 22 anos como moderadamente secos (Figura 1 C). O SPI não considera a classificação pouco seco. $\mathrm{O}$ número de ocorrências de seca estimado pelo SPI foi de 74 meses, dos quais 5 meses para a condição de seca extrema, 29 meses para a classificação muito seco e 40 meses para a seca moderada (Figura 2 C, linha). O SPI apresentou a menor correlação com o PDSI $(0,33)$. O mesmo fato foi observado por Lloyd-Hughes \& Saunders (2002), quando compararam diferentes escalas temporais do SPI (3, 6, 9, 12, 18 e 24 meses) com o PDSI.

O índice RAI foi o que apresentou o maior número de eventos estimados como extremamente secos em 11 anos. Para os eventos classificados como muito secos e moderadamente secos, foram observadas ocorrências nos 26 anos de dados climáticos da série histórica; e, para eventos pouco secos, em 23 anos (Figura 1 D). O número de ocorrências de secas do

Pesq. agropec. bras., Brasília, v.45, n.8, p.771-779, ago. 2010 

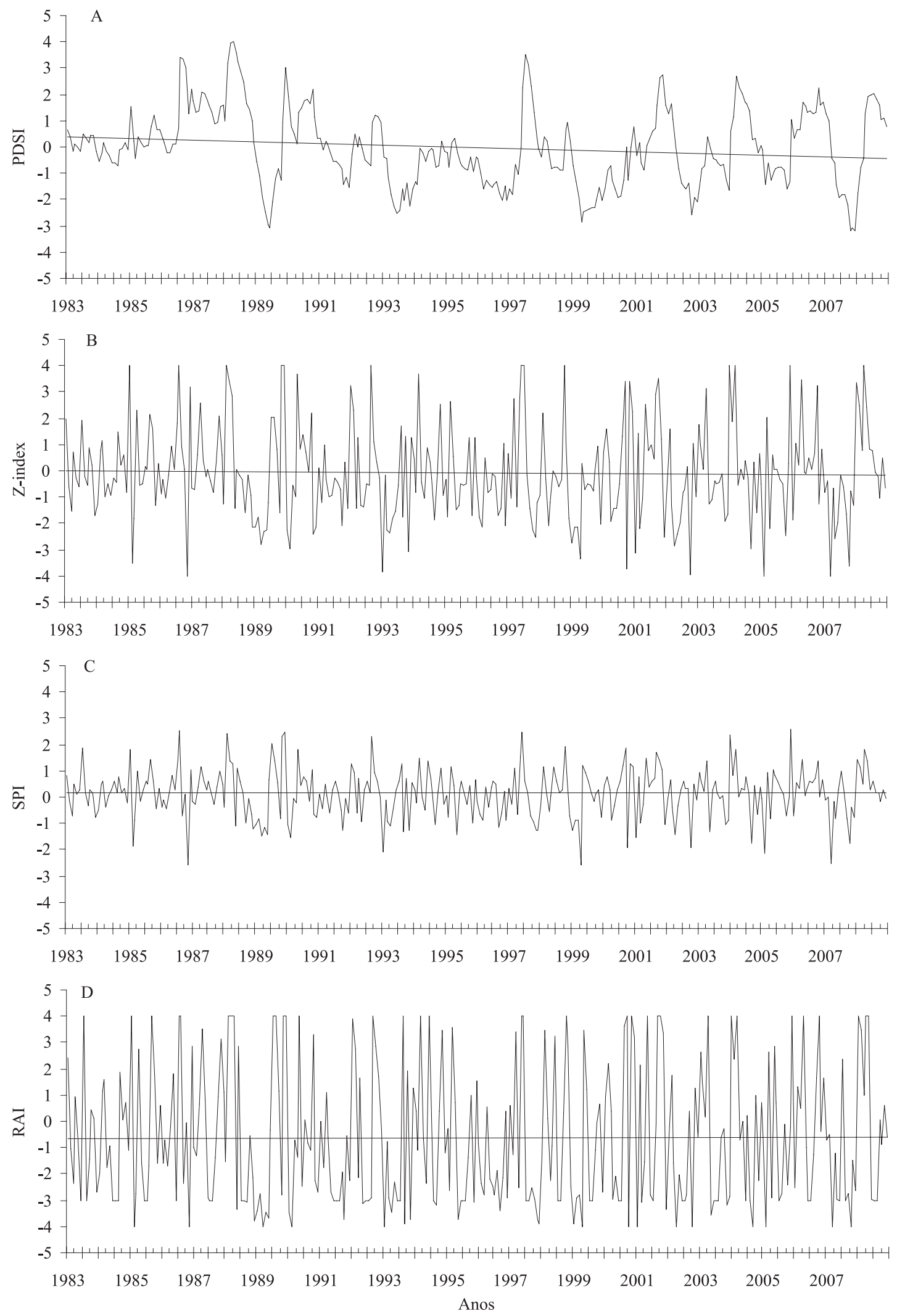

Figura 1. Série temporal dos índices PDSI (A), Z-index (B), SPI (C) e RAI (D), para o Município de Santo Antônio de Goiás, GO. 

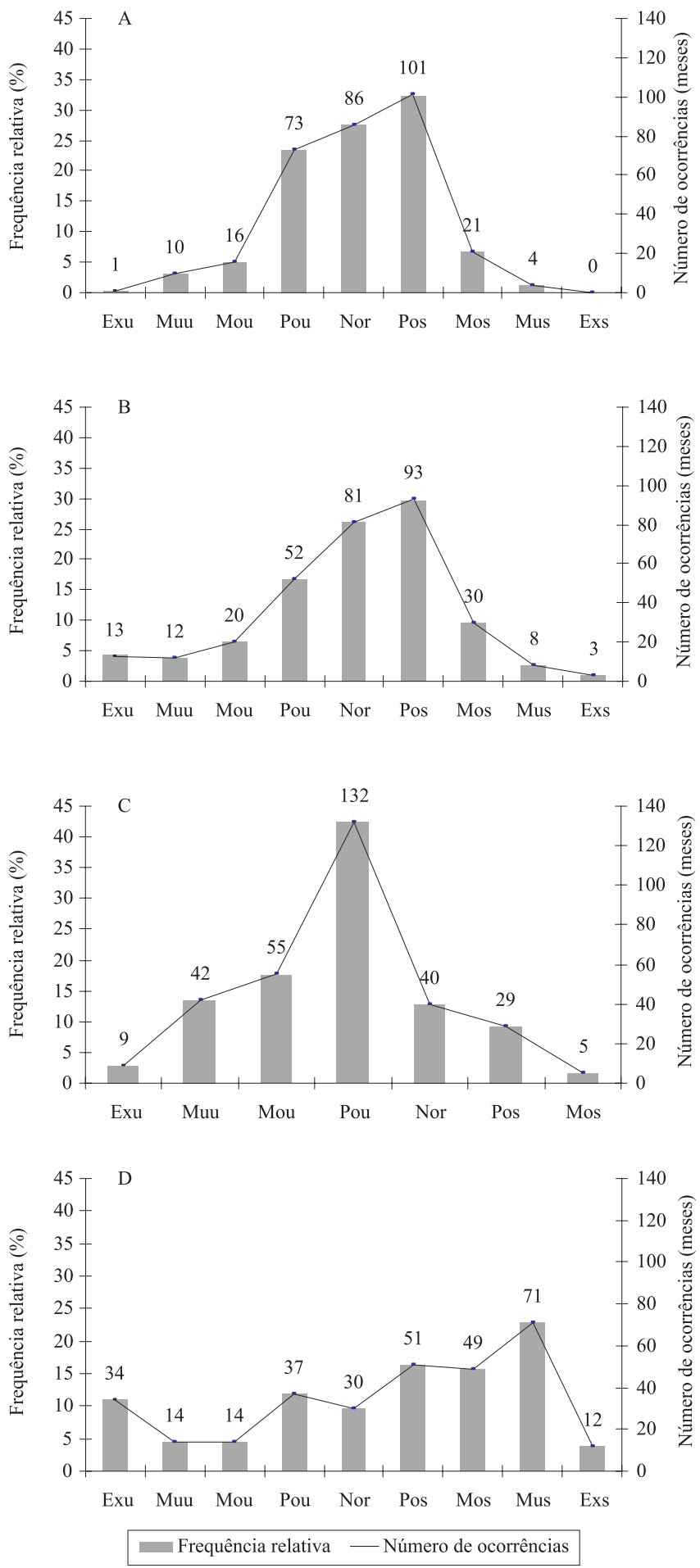

Figura 2. Frequência relativa e número de ocorrências dos valores obtidos no cálculo dos índices de seca PDSI (A), Z-index (B), SPI (C) e RAI (D), em diferentes classes de umidade: Exu, extremamente úmido; Muu, muito úmido; Mou, moderadamente úmido; Pou, pouco úmido; Nor, normal; Pos, pouco seco; Mos, moderadamente seco; Mus, muito seco; Exs, extremamente seco. índice RAI foi de 183 meses, tendo sido observados 12 meses para eventos extremos de seca, 71 meses de eventos tidos como muito secos, 49 meses para eventos moderadamente secos e 51 meses para eventos pouco secos (Figura 2 D, linha). Dos quatro índices analisados, o RAI pode ser considerado o que obteve o maior número de ocorrências de eventos de seca. Isso o classifica como um índice que responde de forma rápida à mudança na quantidade precipitada. Esse índice teve correlação de 0,91 com o Z-index (Tabela 2). Ao contrário do PDSI, os índices Z-index, SPI e RAI não mostraram tendências para o aumento de eventos de seca ao longo do período estudado.

As frequências de ocorrência das classes de umidade dos períodos de acordo com os índices PDSI, SPI e Z-index apresentaram distribuição próxima à normal (Figura 2 A, B e C). Sansigolo (2004) e Morid et al. (2006) observaram o mesmo para os índices SPI e PDSI. Essa característica não foi observada no índice RAI (Figura 2 D). Deve-se salientar que o SPI não considera os grupos de pouca seca/umidade. Isso acarreta maior concentração no grupo normal. Índices que mostram distribuição normal são desejáveis, pois permitem, com maior facilidade, comparações em diferentes localidades (Domingos, 2006).

De acordo com a classificação realizada na produtividade ajustada (diminuição/aumento), observou-se que 13 anos da série histórica apresentaram diminuição na produtividade maior que $2 \%$. O índice acumulado PDSI não representou de forma adequada os anos em que houve diminuição na produtividade (Figura $3 \mathrm{~A}$ ), provavelmente pela falta de ajustes desse índice às condições climáticas da região. Blain \& Brunini (2007a) modificaram os valores parametrizados da equação original do PDSI e relacionaram o início e o fim de um evento de seca em função da razão entre evapotranspiração real e potencial, o que permitiu obter bons resultados na quantificação de secas para o Estado

Tabela 2. Coeficientes de correlação de Pearson entre os índices de seca ${ }^{(1)}$.

\begin{tabular}{lcccc}
\hline & PDSI & Z-index & SPI & RAI \\
\hline PDSI & 1 & 0,44 & 0,33 & 0,32 \\
Z-index & & 1 & 0,91 & 0,87 \\
SPI & & 1 & 0,81 \\
RAI & & & & 1 \\
\hline
\end{tabular}

(1)PDSI, índice de severidade de seca de Palmer; Z-index, índice Z de Palmer; SPI, índice padronizado de precipitação; RAI, índice de anomalia de chuva. 


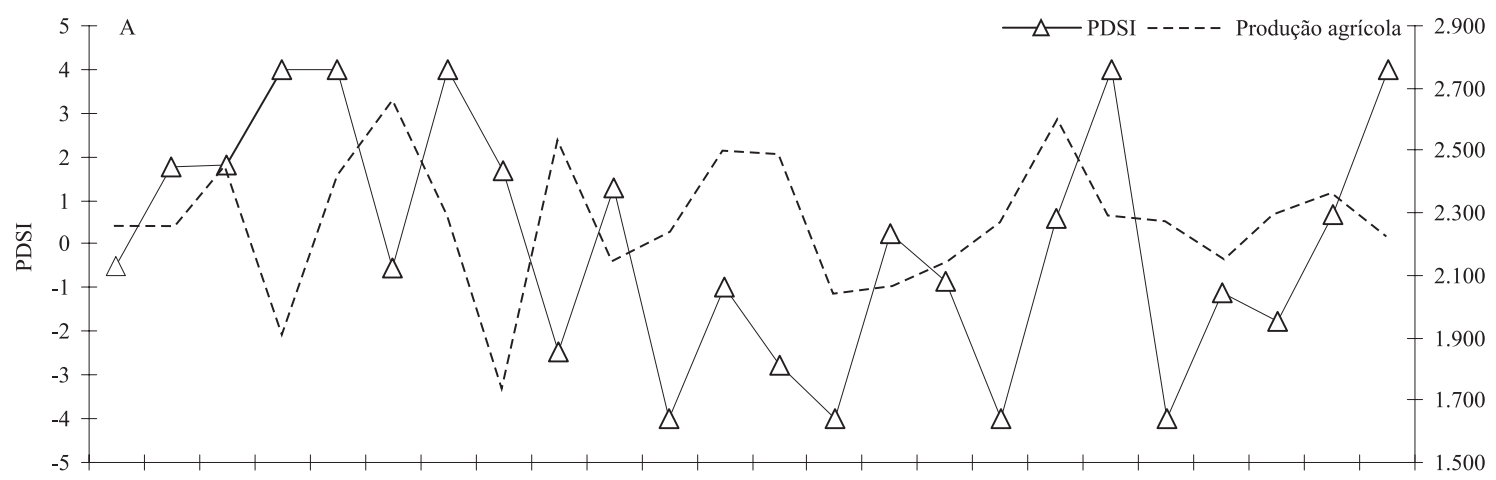

198319841985198619871988198919901991199219931994199519961997199819992000200120022003200420052006

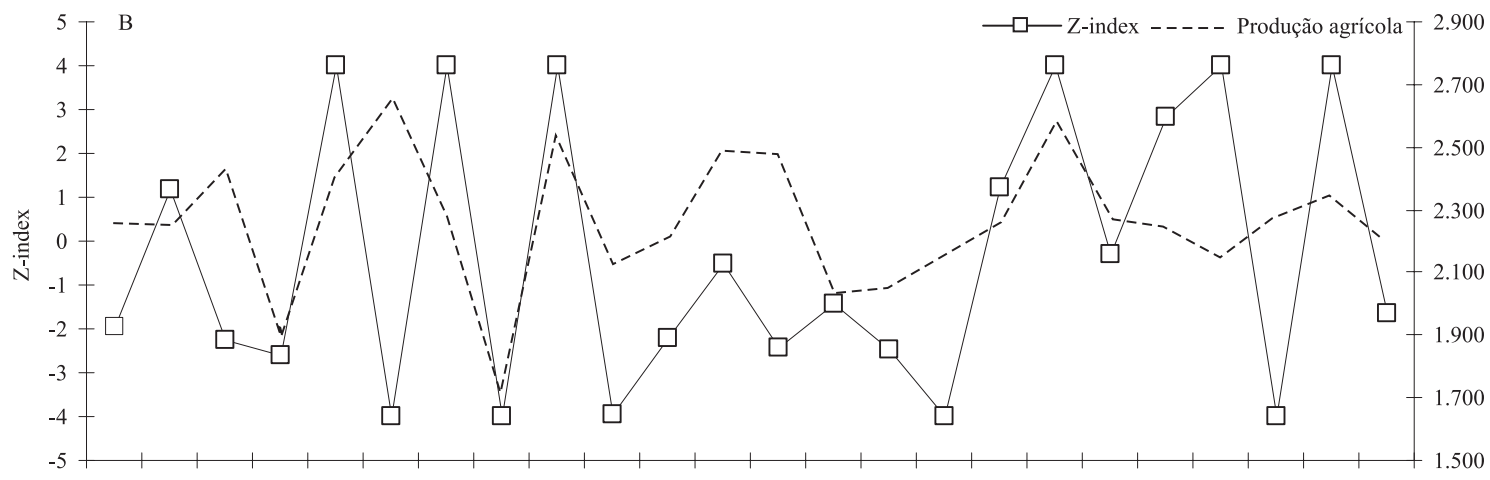

198319841985198619871988198919901991199219931994199519961997199819992000200120022003200420052006

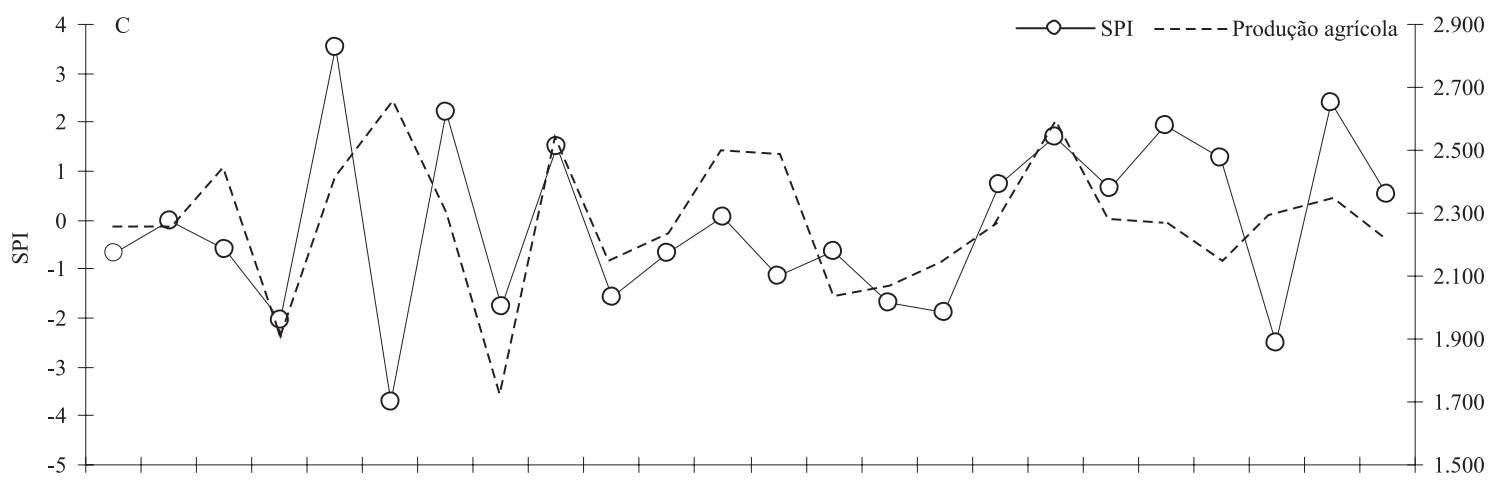

198319841985198619871988198919901991199219931994199519961997199819992000200120022003200420052006

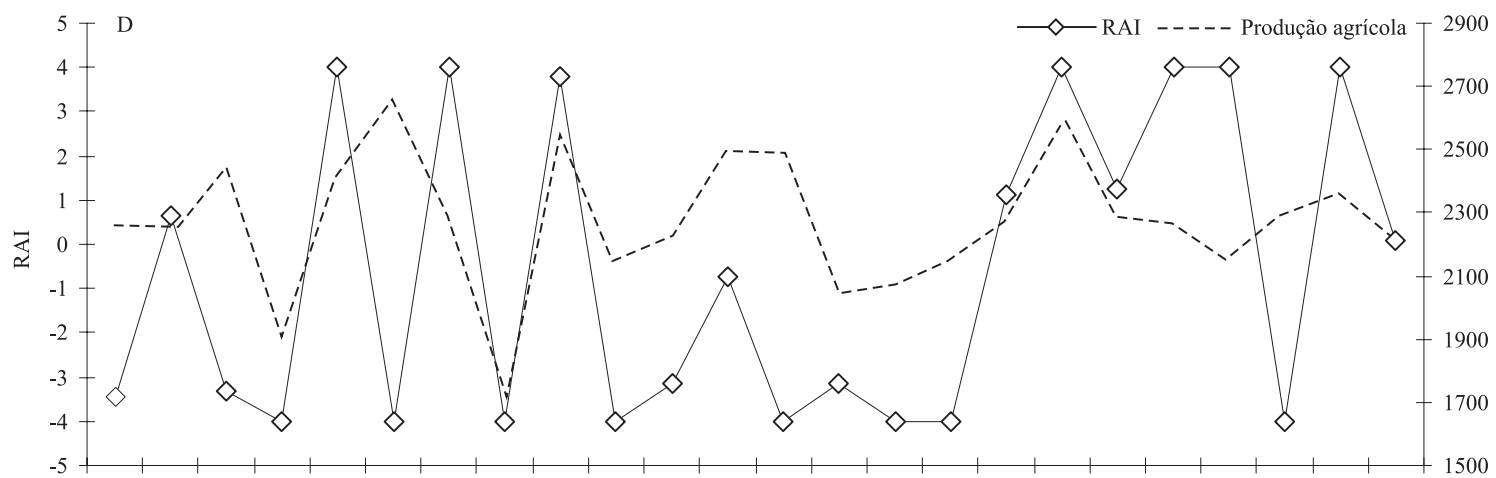

198319841985198619871988198919901991199219931994199519961997199819992000200120022003200420052006 Anos

Figura 3. Comparação dos valores obtidos no cálculo dos índices de seca acumulados PDSI (A), Z-index (B), SPI (C) e RAI (D) com os dados de produtividade ajustada do arroz de terras altas, para a microrregião de Goiânia, GO. 
de São Paulo. Provavelmente, isto seja uma solução para que o PDSI possa ter um melhor desempenho para a região de estudo. $\mathrm{O}$ índice acumulado $\mathrm{Z}$-index mostrou tendência de ajuste mais próximo à produtividade ajustada do arroz de terras altas do que o PDSI (Figura 3 B). Dos 13 anos em que houve diminuição na produtividade ajustada, esse índice acompanhou oito anos com eventos secos. Apesar de o Z-index ser calculado com os mesmos parâmetros do PDSI, ele não leva em consideração as condições de umidade mensais anteriores e, consequentemente, responde de forma rápida às mudanças de umidade. $\mathrm{O}$ índice acumulado SPI e RAI acompanharam as diminuições da produtividade ajustada em 7 dos 13 anos.

Para o ano de 1987/1988, todos os índices indicaram eventos de seca, mas a produtividade ajustada aumentou. Isso pode estar relacionado à não consistência do valor de produtividade para esse ano.

Considerando-se a série histórica da produtividade ajustada, o índice acumulado que apresentou a melhor relação entre eventos de seca/umidade com a produtividade foi o Z-index (Tabela 3); todavia, somente em $54 \%$ dos casos. Para os anos da série histórica da produtividade ajustada em que ela variou mais de $300 \mathrm{~kg} \mathrm{ha}^{-1}$, os índices acumulados que mostraram os melhores números de acertos foram o Z-index e o RAI. Neste cenário, houve aumento no número de acertos para os índices acumulados Z-index, RAI e SPI. Na região sudoeste do Estado de Goiás, os índices SPI

Tabela 3. Percentual de acerto dos índices de seca em relação à série histórica de produtividade ajustada do arroz de terras altas e à série ajustada, considerando-se períodos com variações na produtividade maiores ou menores que $300 \mathrm{~kg} \mathrm{ha}^{-1}$.

\begin{tabular}{|c|c|c|c|}
\hline Indices $^{(1)}$ & $\begin{array}{c}\text { Anos } \\
\text { observados }\end{array}$ & $\begin{array}{c}\text { Anos não } \\
\text { observados }\end{array}$ & $\begin{array}{l}\text { Percentual de } \\
\text { acerto }\end{array}$ \\
\hline & \multicolumn{3}{|c|}{ Série histórica } \\
\hline PDSI & 9 & 15 & 38 \\
\hline Z-index & 13 & 11 & 54 \\
\hline SPI & 12 & 12 & 50 \\
\hline \multirow[t]{2}{*}{ RAI } & 12 & 12 & 50 \\
\hline & \multicolumn{3}{|c|}{ Série ajustada } \\
\hline PDSI & 3 & 6 & 33 \\
\hline Z-index & 7 & 2 & 78 \\
\hline SPI & 6 & 3 & 67 \\
\hline RAI & 7 & 2 & 78 \\
\hline
\end{tabular}

(1)PDSI, índice de severidade de seca de Palmer; Z-index, índice Z de Palmer; SPI, índice padronizado de precipitação; RAI, índice de anomalia de chuva. e Z-index possibilitaram os melhores resultados para quantificar a seca (Faria \& Assunção, 2008).

\section{Conclusões}

1. O índice de anomalia de chuva é o que aponta o maior número de ocorrências de eventos estimados como extremamente secos.

2. Os índices de severidade de seca de Palmer, Z de Palmer e padronizado de precipitação apresentam distribuição normal.

3. O Z-index obteve o melhor desempenho para a microrregião de Goiânia, com base nos critérios número de eventos de seca, distribuição de frequências próxima à normal e número de acertos dos índices de seca em relação à produtividade ajustada do arroz de terras altas.

\section{Agradecimentos}

À Financiadora de Estudos e Projetos, pelo apoio financeiro; ao Conselho Nacional de Desenvolvimento Científico e Tecnológico, por concessão de bolsa.

\section{Referências}

BLAIN, G.C.; BRUNINI, O. Análise comparativa dos índices de seca de Palmer, Palmer adaptado e índice padronizado de precipitação no Estado de São Paulo. Revista Brasileira de Meteorologia, v.22, p.105-111, 2007a.

BLAIN, G.C.; BRUNINI, O. Análise da escala temporal de monitoramento das secas agrícolas e meteorológicas no Estado de São Paulo. Revista Brasileira de Meteorologia, v.22, p.255-261, 2007b.

CLEVELAND, W.S. Robust locally weighted regression and smoothing scatterplots. Journal of the American Statistical Association, v.74, p.829-836, 1979.

DOMINGOS, S.I.S. Análise do índice de seca standardized precipitation index (SPI) em Portugal Continental e sua comparação com o Palmer drought severity index (PDSI). 2006. 62p. Tese (Licenciatura) - Universidade de Lisboa, Portugal.

DUNKEL, Z. Brief surveying and discussing of drought indices used in agricultural meteorology. Idõjárás, v.113, p.23-37, 2009.

FARIA, S.M.; ASSUNÇÃO, H.F. da. Estimativa dos índices de seca PDSI e Z de Palmer e avaliação das suas influências sobre a produção agrícola no Município de Jataí - GO. Revista Geografia, Ensino \& Pesquisa, v.11, p.3736-3749, 2008.

FERNANDES, D.S.; HEINEMANN, A.B.; PAZ, R.L.F. da; AMORIM, A. de O.; CARDOSO, A.S. Índices para a quantificação da seca. Santo Antônio de Goiás: Embrapa Arroz e Feijão, 2009. 45p. (Embrapa Arroz e Feijão. Documentos, 244). 
FREITAS, M.A. de S. Um sistema de suporte à decisão para o monitoramento de secas meteorológicas em regiões semi-áridas. Revista Tecnologia, p.84-95, 2005. Suplemento.

GARAGORRY, F.L.; REGO, A.M. AGROTEC: base relacional de dados estatísticos: estrutura de dados. Versão 1.0. Brasília: Embrapa-CPAC, 1997. 20p.

GUTTMAN, N.B. Comparing the Palmer drought index and the standardized precipitation index. Journal of the American Water Resources Association, v.34, p.113-121, 1998.

HEIM, R.R. A review of twentieth: century drought indices used in the United States. Bulletin of the American Meteorology Society, v.83, p.1149-1163, 2002.

HEINEMANN, A.B.; SILVA, S.C. da; LOPES JUNIOR, S.; AMORIM, A. de O.; ANDRADE, C. de L.T. de; BASTOS, E.A.; PAZ, R.L.F. da. Características climáticas dos municípios de Santo Antônio de Goiás (GO), Porangatu (GO), Janaúba (MG), Sete Lagoas (MG), Parnaíba (PI) e Teresina (PI), Brasil. Santo Antônio de Goiás: Embrapa Arroz e Feijão. 2007. 36p. (Embrapa Arroz e Feijão. Documentos, 214).

HOLLINGER, S.E.; EHLER, E.J.; CARLSON, R.E. Midwestern United States corn and soybean yield response to changing El Niño-Southern Oscillation conditions during the growing season. In: HATFIELD, J.L.; VOLENEC, J.J.; DICK, W.A.; KRAL, D.M. (Ed.). Impacts of EI Niño and climate variability on agriculture. Madison: American Society of Agronomy, 2001. (ASA. Special publication, 63).

INSTITUTO BRASILEIRO DE GEOGRAFIA E ESTATÍSTICA. Levantamento sistemático da produção agrícola: 2009. Disponível em: <www.ibge.gov.br/home/estatistica/indicadores/ agropecuaria/lspa/>. Acesso em: 22 jan. 2010.

KEYANTASH, J.; DRACUP, J.A. The quantification of drought: an evaluation of drought indices. Bulletin of the American Meteorology Society, v.83, p.1167-1180, 2002.

LLOYD-HUGHES, B.; SAUNDERS, M.A. A drought climatology for Europe. International Journal of Climatology, v.22, p.1571-1592, 2002.

LOUKAS, A.; VASILIADES, L. Probabilistic analysis of drought spatiotemporal characteristics in Thessaly region, Greece. Natural Hazards and Earth System Sciences, v.4, p.719-731, 2004.

MACEDO, M.J.H.; GUEDES, R.V. de S.; SOUZA, F. de A.S. de; DANTAS, F.R. da C. Análise do índice padronizado de precipitação para o Estado da Paraíba, Brasil. Revista Ambiente \& Água, v.5, p.204-214, 2010.

MCKEE, T.B.; DOESKEN, N.J.; KLEIST, J. The relationship of drought frequency and duration to the time scales. In: CONFERENCE ON APPLIED CLIMATOLOGY, 8., 1993, Anaheim. Anais. Boston: American Meteorological Society, 1993. p.179-184.

MORID, S.; SMAKHTIN, V.; MOGHADDASI, M. Comparison of seven meteorological indices for drought monitoring in Iran. International Journal of Climatology, v.26, p.971-985, 2006.

NTALE, H.K.; GAN, T.Y. Drought indices and their application to East Africa. International Journal of Climatology, v.23, p.1335-1357, 2003.
PALMER, W.C. Meteorological drought. Washington: U.S. Department of Commerce, 1965. 58p. (U.S. Department of Commerce. Research paper, 45).

PARK, S.; FEDDEMA, J.J.; EGBERT, S.L. MODIS land surface temperature composite data and their relationships with climatic water budget factors in the Central Great Plains. International Journal of Remote Sensing, v.26, p.1127-1144, 2005.

PINHEIRO, B. da S. Características morfológicas da planta relacionadas a produtividade. In.: SANTOS, A.B. dos; STONE, L.F.; VIEIRA, N.R. de A. (Ed.). A cultura do arroz no Brasil. 2.ed. rev. Santo Antônio de Goiás: Embrapa Arroz e Feijão, 2006. 1000p.

QUIRING, S.M. Developing objective operational definitions for monitoring drought. Journal of Applied Meteorology and Climatology, v.48, p.1217-1229, 2009a.

QUIRING, S.M. Monitoring drought: an evaluation of meteorological drought indices. Geography Compass, v.3, p.64-88, 2009 b.

REDMOND, K.T. The depiction of drought: a commentary. Bulletin of the American Meteorological Society, v. 83, p.1143-1147, 2002.

ROOY, M.P. van. A rainfall anomaly index independent of time and space. Notos, v.14, p.1-43, 1965.

SALAS, J.D.; FU, C.J.; CANCELLIERE, A.; DUSTIN, D.; BODE, D.; PINEDA, A.; VINCENT, E. Characterizing the severity and risk of drought in the Poudre River, Colorado. Journal of Water Resources Planning and Management, v.131, p.383-393, 2005.

SANSIGOLO, C.A. Análise comparativa do desempenho dos índices de seca de Palmer (PDSI) e da precipitação normalizada (SPI) em Piracicaba, SP (1917-2001). Revista Brasileira de Meteorologia, v.19, p.237-242, 2004.

SCHAFFERT, R.E.; ALVES, V.M.C.; PARENTONI, S.N.; RAGHTHAMA, K.G. Genetic control of phosphorus uptake and utilization efficiency in maize and sorghum under marginal soil conditions. In: RIBAUT, J.-M.; POLAND, D. (Ed.). Molecular approaches for the genetic improvement of cereals for stable production in water-limited environments: a strategic planning workshop held at CIMMYT. Mexico: CIMMYT, 2000. p.79-85.

SILVA, D.F. da; SOUZA, F. de A.S.; KAYANO, M.T. Uso de IAC e ondeletas para análise da influência das multi-escalas temporais na precipitação da Bacia do rio Mundaú. Engenharia Ambiental, v.6, p.180-105, 2009.

THOM, H.C.S. A note on the gamma distribution. Monthly Weather Review, v.86, p.117-122, 1958.

THORNTHWAITE, C.W.; MATHER, J.R. The water balance. Climatology, v.8, p.1-40, 1955.

WILHITE, D.A. Drought. In: HOLTON, J.A.; PYLE, C.; CURRY, J.A. (Ed.). Encyclopedia of atmospheric science. New York: Elsevier, 2003. p.650-658.

WU, H.; SVOBODA, M.D.; HAYES, M.J.; WILHITE, D.A.; WEN, F. Appropriate application of the standardized precipitation index in arid locations and dry seasons. International Journal of Climatology, v.27, p.65-79, 2005.

Recebido em 1 de maio de 2010 e aprovado em 13 de julho de 2010 